\title{
NOMBRE DE CAS CONFIRMÉS D'HÉPATITE VIRALE AU BRÉSIL ENTRE 2010 ET 2015
}

\section{ARTICLE ORIGINAL}

NUNES, Filipe Sales', FACCO, Lucas², FECURY, Amanda Alves³, ARAÚJO, Maria Helena Mendonça de ${ }^{4}$, OLIVEIRA, Euzébio de ${ }^{5}$, DENDASCK, Carla Viana ${ }^{6}$, SOUZA, Keulle Oliveira da ${ }^{7}$, DIAS, Claudio Alberto Gellis de Mattos $^{8}$

NUNES, Filipe Sales. Et al. Nombre de cas confirmés d'hépatite virale au Brésil entre 2010 et 2015. Revista Científica Multidisciplinar Núcleo do Conhecimento. An 05, Ed. 11, vol. 25, p. 71-80. novembre 2020. ISSN: 2448-0959, Lien d'accès: https://www.nucleodoconhecimento.com.br/sante/dhepatite-virale, DOI: 10.32749/nucleodoconhecimento.com.br/sante/dhepatite-virale

\section{RÉSUMÉ}

L'hépatite virale est une maladie infectieuse qui attaque le foie, et ses agents causatous sont des virus. Cette étude vise à démontrer le nombre de cas confirmés d'hépatite virale au Brésil entre 2010 et 2015. Une recherche a été effectuée dans la base de données DATASUS sur le site Web (http://datasus.saude.gov.br/). L'hépatite représente un vaste problème de santé publique au Brésil. Chez les personnes infectées, une grande partie est composée de personnes de sexe masculin, et la baisse visible de la demande de services de santé est un facteur important pour

\footnotetext{
${ }^{1}$ Technicien en réseaux informatiques, diplômé de l'Institut fédéral d'Amapá (IFAP).

2 Étudiant du cours de médecine de l'Université fédérale d'Amapá (UNIFAP).

${ }^{3}$ Biomédical, Docteur en maladies tropicales, Professeur et chercheur du Cours médical de l'Université fédérale d'Amapá (UNIFAP).

${ }^{4}$ Médecin, professeur et chercheur du cours de médecine de l'Université fédérale d'Amapá (UNIFAP).

${ }^{5}$ Biologiste, $\mathrm{PhD}$ en maladies topiques, professeur et chercheur du cours d'éducation physique de l'Université fédérale du Pará (UFPA).

${ }^{6}$ Théologien, Docteur en psychanalyse, chercheur au Centre de Recherche et Études avancées - CEPA.

${ }^{7}$ Sociologue, étudiant à la maîtrise en études anthropiques en Amazonie, membre du groupe de recherche "Laboratoire d'éducation, d'environnement et de santé » (LEMAS/UFPA).

${ }^{8}$ Biologiste, PhD en théorie et recherche comportementale, professeur et chercheur du Diplôme d'études supérieures en éducation professionnelle et technologique (PROFEPT), Institut fédéral d'Amapá (IFAP).
}

RC: 67661

Disponible en: https://www.nucleodoconhecimento.com.br/sante/dhepatite-virale 
cette constatation. Les hépatites $B$ et $C$ sont les plus courantes chez les hépatites virales et l'un des facteurs importants et contributifs du taux de virus de l'hépatite infecté est leur co-infection par le VIH. Des tests de laboratoire (immunoassay, tests moléculaires) devraient être effectués pour détecter les marqueurs et déterminer l'agent étiologique à l'origine de la pathologie.

Mots-clés: Épidémiologie, virus, hépatite.

\section{INTRODUCTION}

L'hépatite virale est une maladie infectieuse qui attaque le foie. Ils sont considérés comme un problème de santé publique au Brésil en raison de leur grand nombre de cas confirmés (MARGREITER et al., 2015; LEITE, et al., 2020).

Les agents étiologiques découverts de l'hépatite virale sont les virus HAV, HBV, VHC, VHD et VHE, qui ont en commun la facilité de liaison avec les cellules hépatiques. Ils présentent des différences dans leur forme clinique et leurs caractéristiques épidémiologiques (NUNES et al., 2016).

Les hépatites $A(V H A)$ et $E(V H E)$ ont leurs virus transmis par voie fécale-orale, et peuvent être trouvés dans l'eau et les aliments contaminés. Le manque de traitement adéquat de l'eau consommée et la manipulation inadéquate des aliments sont les principaux facteurs de propagation du virus. La maladie, lors de la présentation des symptômes, peut causer une diminution de l'appétit, de la fièvre et des changements dans la couleur de l'urine de l'individu infecté (MOCBEL et al., 2016).

Le virus de l'hépatite $A$ a un vaccin efficace, considéré comme sûr, et peut maintenir une immunité de plusieurs de 5 à 10 ans. Le vaccin est divisé en deux doses et peut être appliqué aux enfants dès la première année (FERREIRA et al., 2014). L'hépatite E a un vaccin, mais pas à l'échelle mondiale. Initialement commercialisé en 2012 et produit en Chine, il est déjà considéré comme efficace (NUNES et al., 2016).

RC: 67661

Disponible en: https://www.nucleodoconhecimento.com.br/sante/dhepatite-virale 
Le virus de l'hépatite $\mathrm{C}(\mathrm{VHC})$ se transmet principalement par transfusion sanguine, ainsi que par des rapports sexuels, des formes congénitales et le partage d'objets tranchants ou d'hygiène personnelle. L'hépatite $\mathrm{C}$ a un diagnostic plus compliqué en raison de la complexité de son virus. Dans moins de la moitié des cas, il n'est pas possible d'identifier la source mécanique de l'infection. La plupart des cas d'hépatite C atteignent la forme chronique sans présenter de symptômes. Ils ne se manifestent habituellement qu'après des années d'infection lorsque les individus se retrouvent à un stade plus grave. La minorité de cas progresse habituellement à la cirrhose de foie ou au cancer du foie (GUSMÃO et autres, 2017).

Le traitement varie selon et le génotype du virus, en utilisant des médicaments pour prévenir la reproduction du virus, réduisant ainsi l'aggravation de l'infection. La durée du traitement peut être de 48 à 72 semaines, où il peut y avoir une diminution énorme de la charge virale chez le patient, mais pas une extinction totale du virus (SILVA et al., 2014). Un vaccin contre le virus de l'hépatite C n'a pas encore été mis au point. L'un des moyens de prévenir la propagation du virus est la mobilisation de groupes à risque, tels que les usagers de drogues et les professionnels de la santé (GUSMÃO et al., 2017).

L'hépatite $B(\mathrm{VHB})$ voit son virus transmis verticalement, transmis de la mère à l'enfant au moment de l'accouchement ou de l'allaitement. Aussi en partageant des objets pointus tels que des aiguilles et des pinces, transfusion sanguine et forme sexuelle (FRANCISCO et al., 2015).

Les hépatites $B$ et $D$ peuvent se présenter sous deux formes cliniques : aiguë et chronique. Lorsqu'ils présentent des symptômes, ils sont identiques, comme un malaise physique; changements dans la coloration de l'urine; excréments; jaunâtre de la peau et des yeux (BRASIL, 2014; BRASIL, 2014a).

Le virus de l'hépatite $D(V H D)$ exige directement que le virus de l'hépatite $B(V H B)$ soit infectieux, de sorte que les moyens de transmission sont les mêmes, puisqu'il n'est pas possible pour une personne d'être infectée par le VHB sans VHB. Le 
vaccin contre le virus de l'hépatite $B$ est également efficace pour la vaccination contre l'hépatite $D$ en raison de la relation entre l'infection par le virus articulaire (NUNES et al., 2016).

\section{BUT}

Démontrer le nombre de cas confirmés d'hépatite virale au Brésil entre 2010 et 2015.

\section{MÉTHODE}

Une recherche a été effectuée dans la base de données DATASUS sur le site (http://datasus.saude.gov.br/), d'où les données ont été supprimées, suivant les étapes suivantes: Dans le menu du site, nous avons choisi l'onglet "Accès à l'information » puis l'option « HEALTH INFORMATION TABNET », puis l'option «Épidémiologies et morbidité ». Après le chargement de la page, le groupe «Maladies et maladies de notification- À partir de 2007 (SINAN) 》 a été sélectionné et l'option «Hépatite » a été sélectionnée et dans l'onglet «Couverture géographique » l'option «Brésil par région, UF et municipalité » a été sélectionnée.Dans l'option colonne que vous avez sélectionnée, «Non actif », «Sexe », «Groupe d'âge », « Classe. Étiologique », « Source Mecan Infection », «Classe. Finale», «Forme clinique », «Scolarisation». Pour chaque élément sélectionné dans la colonne, dans la ligne toujours «Année Diag / symptômes », et dans la période des années 2010 à 2015 ont toujours été utilisés. Les données ont été compilées dans l'application Excel, un composant de la suite Microsoft Corporation Office. La recherche bibliographique a été effectuée dans des articles scientifiques, à l'aide d'ordinateurs du laboratoire informatique de l'Institut fédéral d'éducation, de science et de technologie d'Amapá, campus de Macapá, situé à: Rodovia BR 210 KM 3, s/n - Bairro Brasil Novo. CEP: 68.909-398, Macapá, Amapá, Brasil.

$\mathrm{RC}: 67661$

Disponible en: https://www.nucleodoconhecimento.com.br/sante/dhepatite-virale 


\section{RÉSULTATS}

La figure 1 montre le nombre de cas confirmés d'hépatite virale au Brésil entre 2010 et 2015. Entre 2010 et 2013, les données montrent une augmentation du nombre de cas. Entre 2013 et 2015, il y a une diminution des infections virales à l'hépatite.

Figure 1 Nombre de cas confirmés d'hépatite virale au Brésil entre 2010 et 2015.

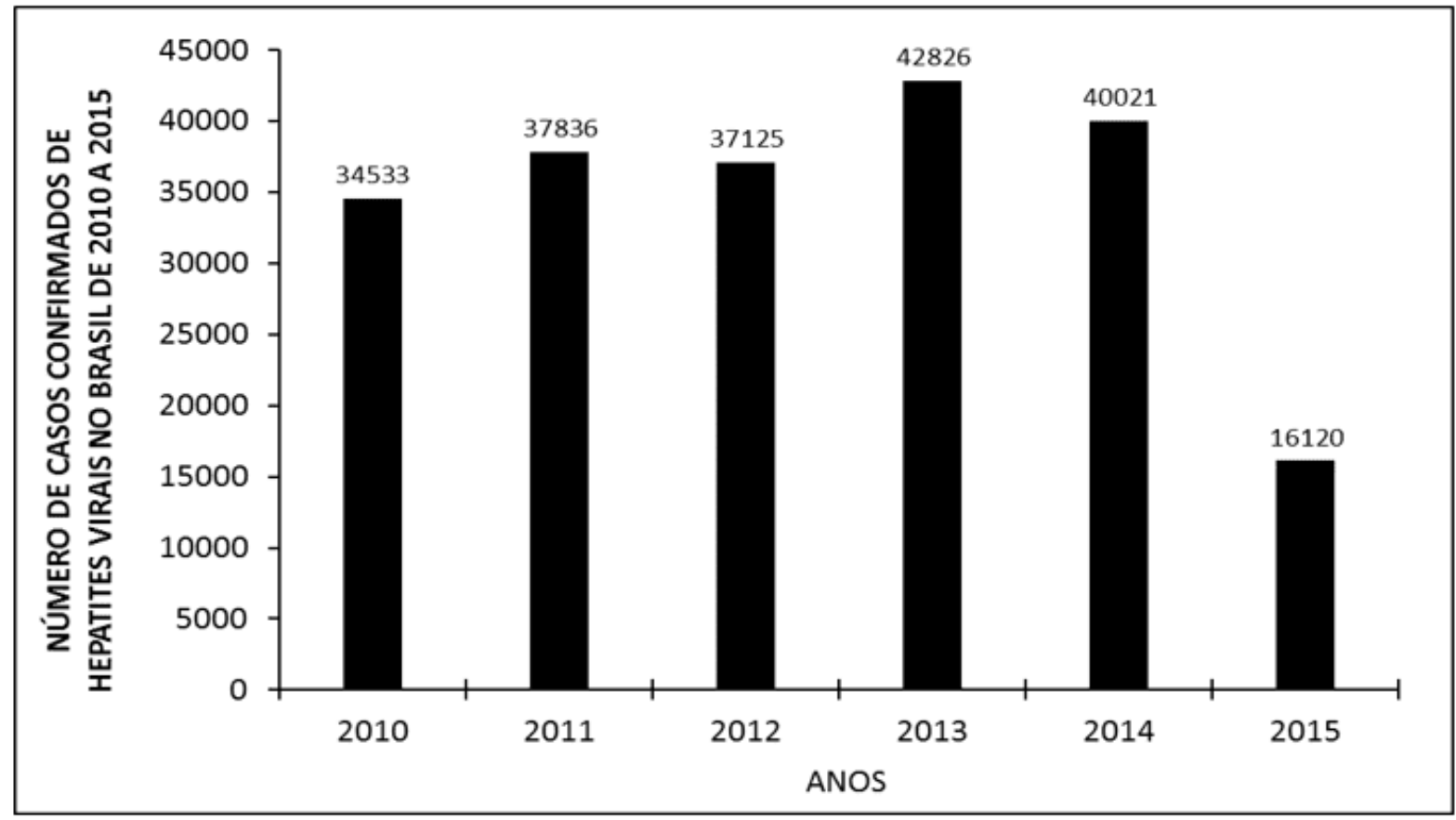

La figure 2 montre le nombre de cas confirmés d'hépatite virale au Brésil entre 2010 et 2015 selon le sexe. Les données montrent un plus grand nombre de cas chez les hommes par rapport aux femmes.

RC: 67661

Disponible en: https://www.nucleodoconhecimento.com.br/sante/dhepatite-virale 
Figure 2 Nombre de cas confirmés d'hépatite virale au Brésil entre 2010 et 2015 selon le sexe.

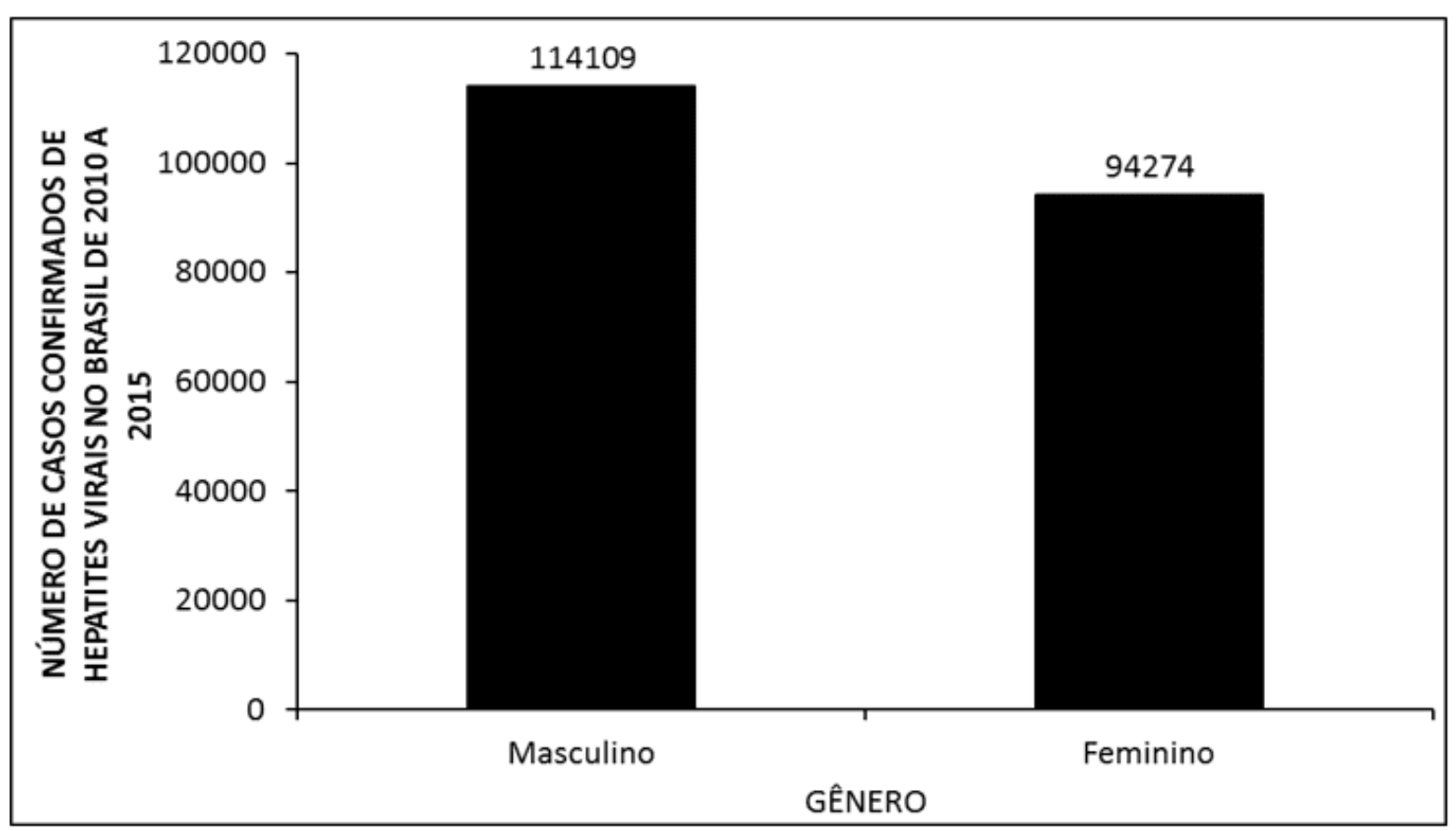

La figure 3 montre le nombre de cas confirmés d'hépatite virale au Brésil entre 2010 et 2015 selon le groupe d'âge. Le plus grand nombre de cas se produit dans le groupe d'âge entre 40 et 59 ans, et le deuxième plus grand nombre entre 20 et 39 ans.

RC: 67661

Disponible en: https://www.nucleodoconhecimento.com.br/sante/dhepatite-virale 
Figure 3 Nombre de cas confirmés d'hépatite virale au Brésil entre 2010 et 2015 selon le groupe d'âge

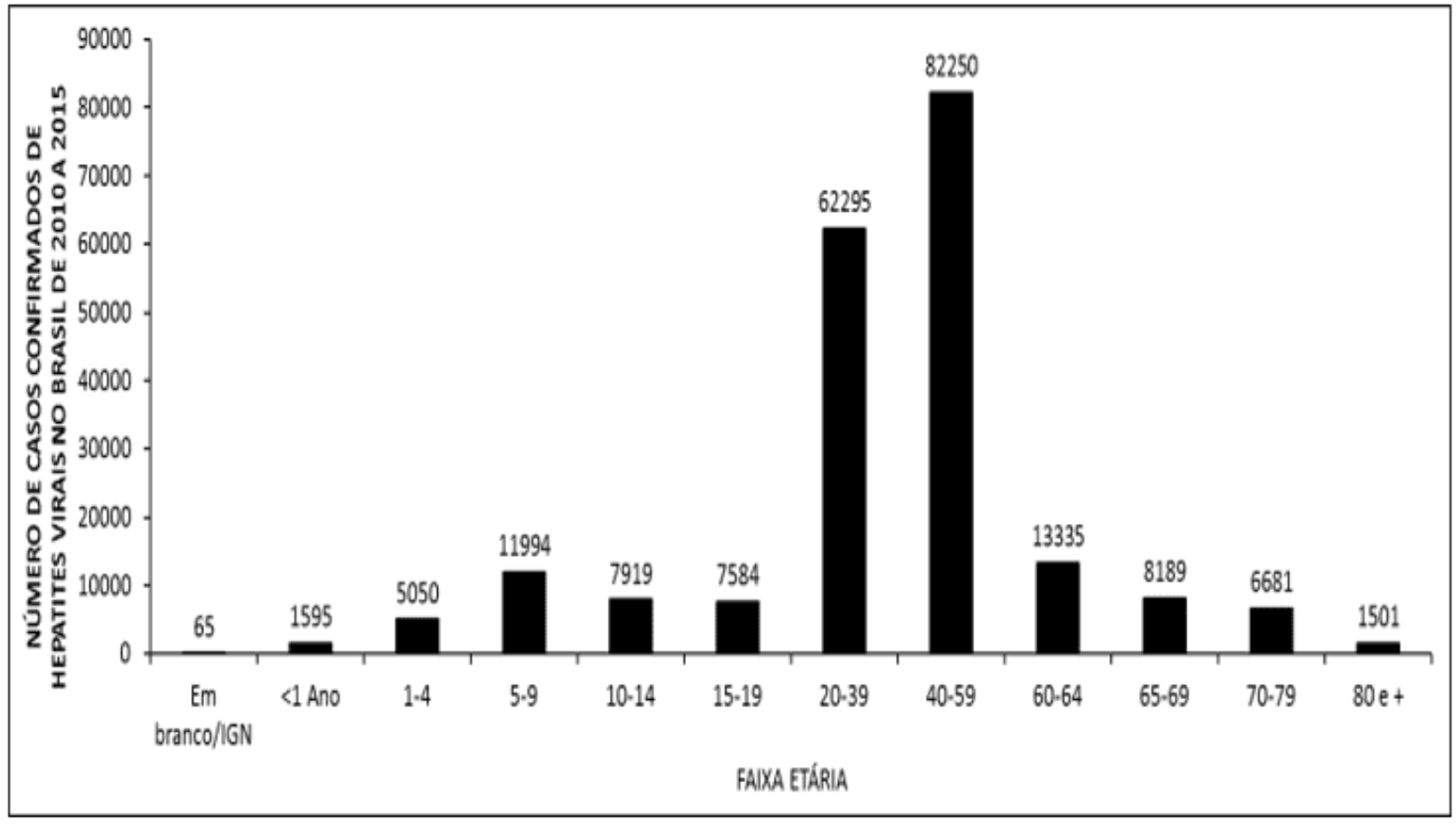

La figure 4 montre le nombre de cas confirmés d'hépatite virale au Brésil entre 2010 et 2015 par classe étiologique, le taux le plus élevé de la maladie se produisant avec la présentation du virus $\mathrm{C}$ le deuxième plus important avec le virus $\mathrm{B}$.

RC: 67661

Disponible en: https://www.nucleodoconhecimento.com.br/sante/dhepatite-virale 
Figure 4 Nombre de cas confirmés d'hépatite virale au Brésil de 2010 à 2015 par classe étiologique.

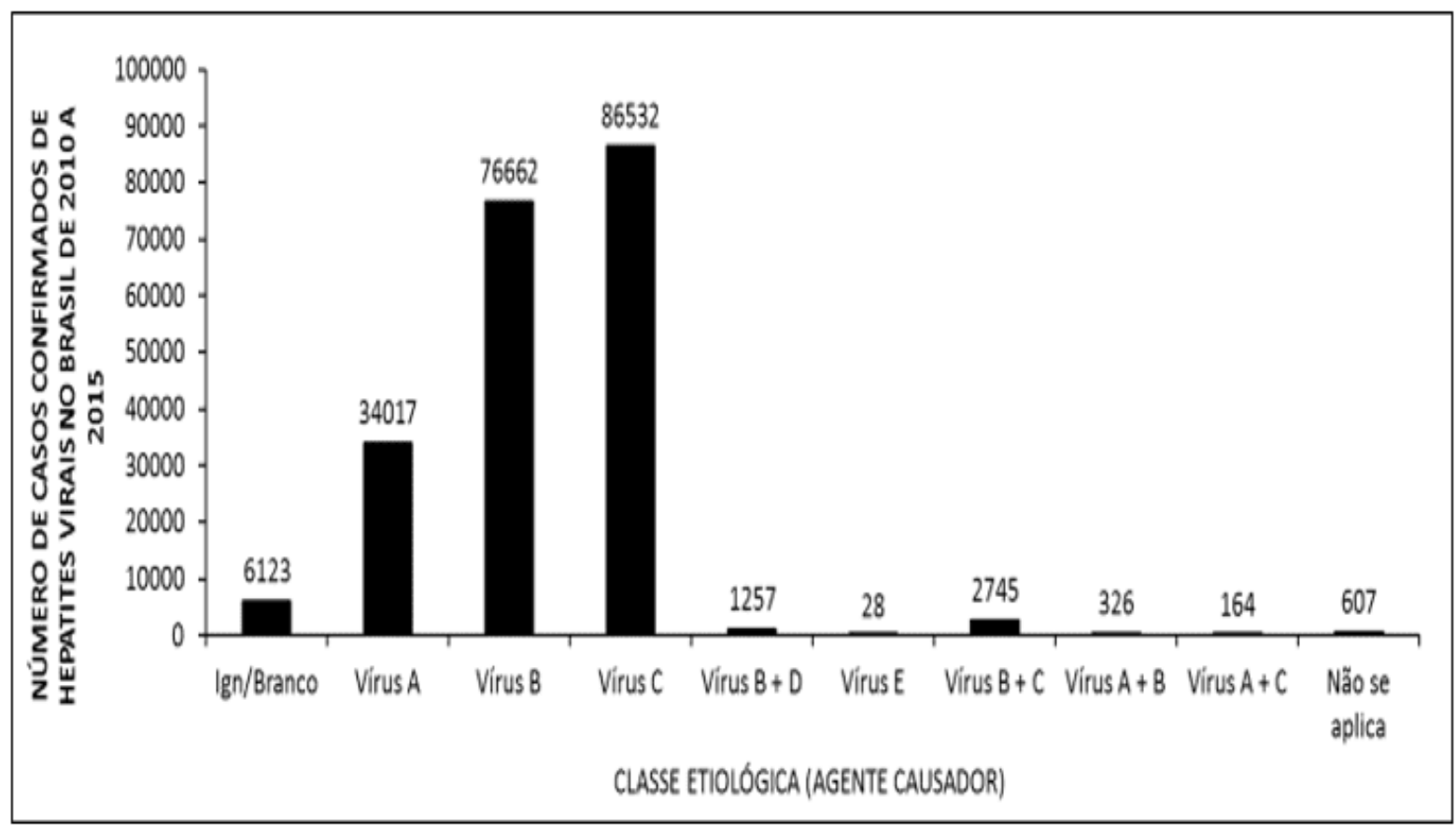

La figure 5 montre le nombre de cas confirmés d'hépatite virale au Brésil entre

2010 et 2015 par source mécanique de contamination, ce qui démontre que le plus grand nombre de cas, où la source mécanique est reconnue, a été par transmission sexuelle et deuxième par Food/Water.

RC: 67661

Disponible en: https://www.nucleodoconhecimento.com.br/sante/dhepatite-virale 
Figure 5 Graphique avec le nombre de cas confirmés d'hépatite virale au Brésil de 2010 à 2015 par source mécanique.

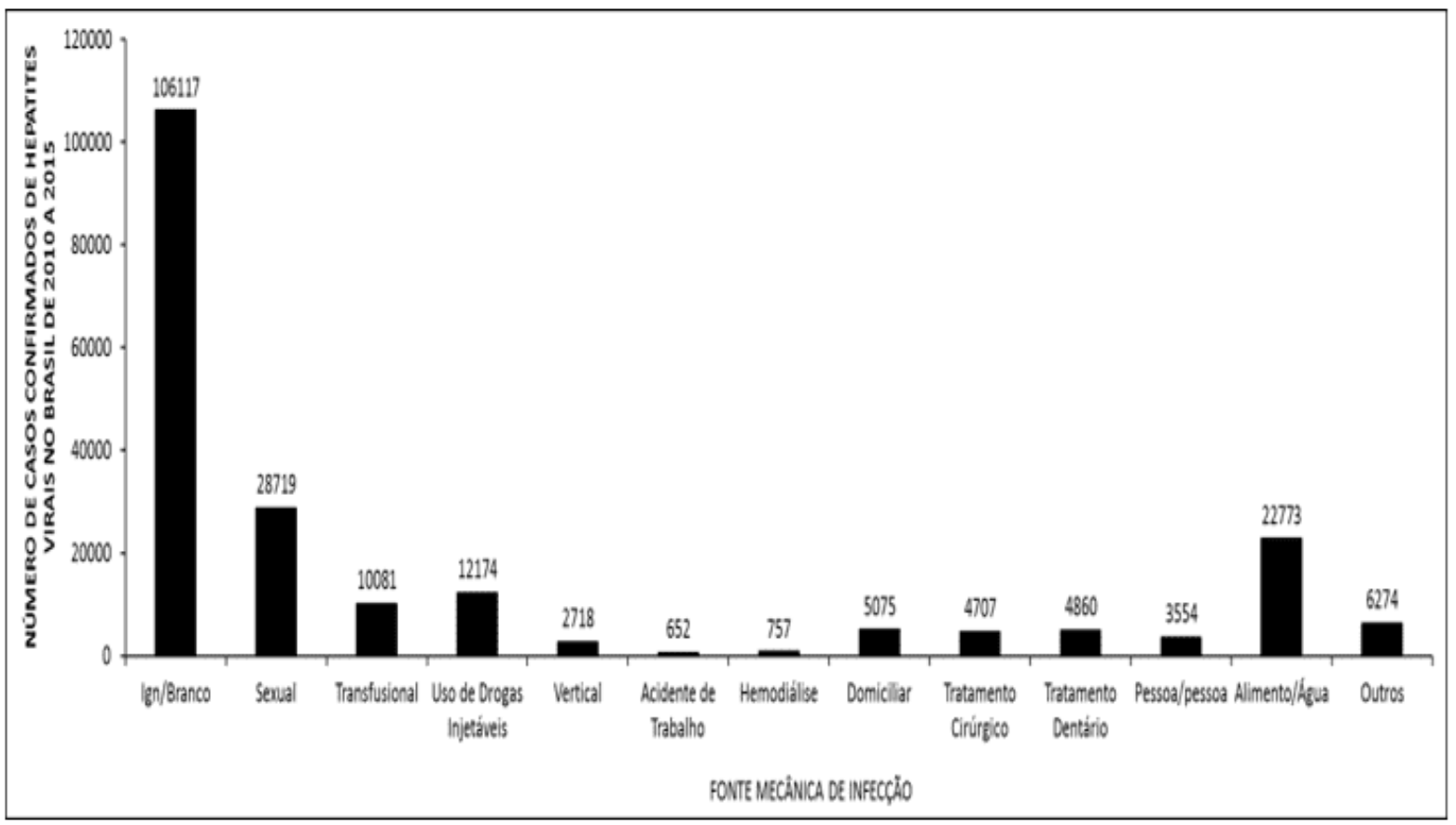

La figure 6 montre le pourcentage de cas confirmés d'hépatite virale au Brésil entre 2010 et 2015 selon sa classification finale. II a été enregistré que le pourcentage le plus élevé de diagnostics a été effectué d'une manière de laboratoire.

RC: 67661

Disponible en: https://www.nucleodoconhecimento.com.br/sante/dhepatite-virale 
Figure 6 Graphique avec pourcentage de cas confirmés d'hépatite virale au Brésil par classification finale entre 2010 et 2015.

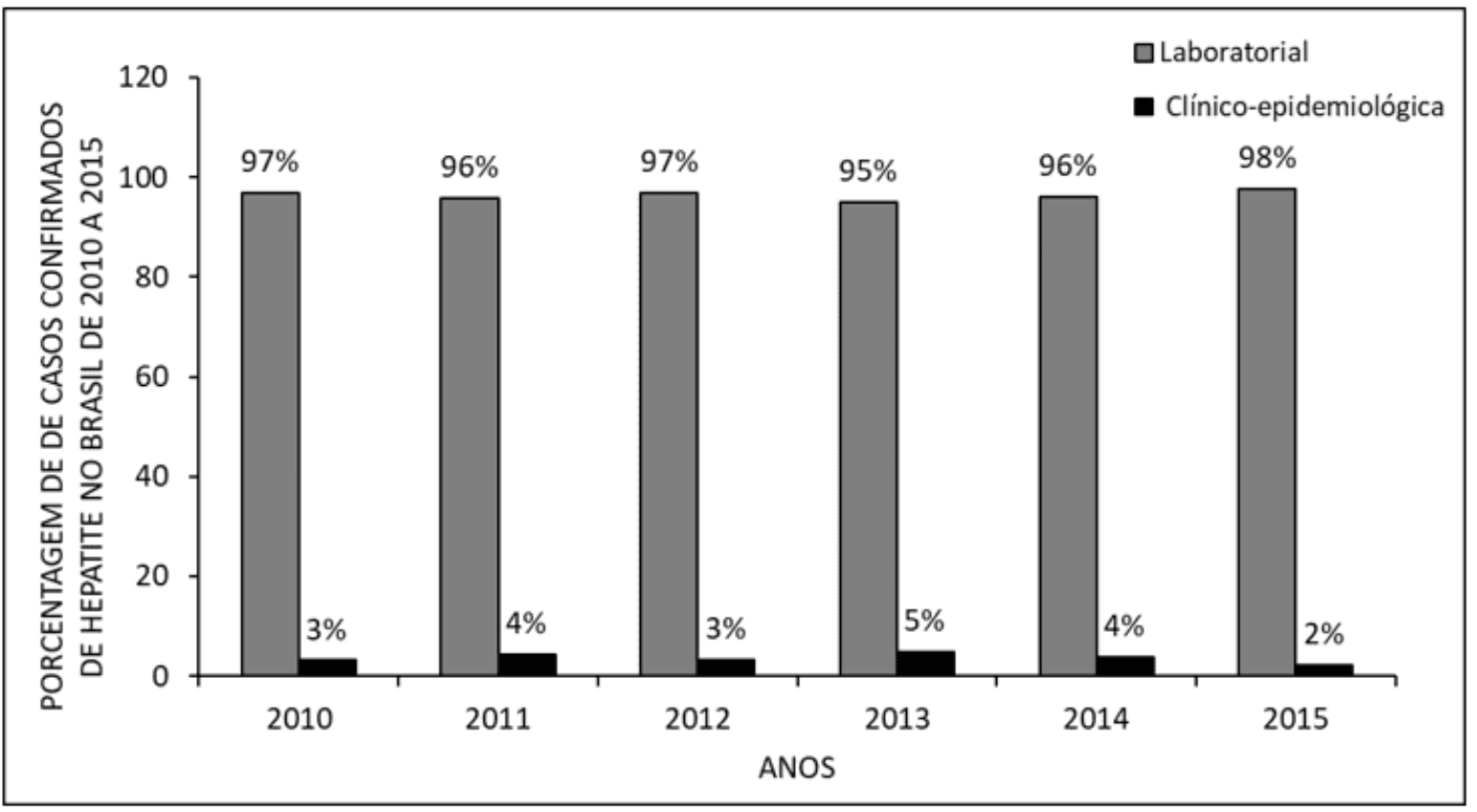

La figure 7 montre le nombre de cas confirmés d'hépatite virale au Brésil par forme clinique entre 2010 et 2015 , ce qui démontre que l'incidence la plus élevée se présente sous forme d'hépatite/porteur chronique.

RC: 67661

Disponible en: https://www.nucleodoconhecimento.com.br/sante/dhepatite-virale 
Figure 7 Nombre de cas confirmés d'hépatite virale au Brésil de 2010 à 2015 sous forme clinique

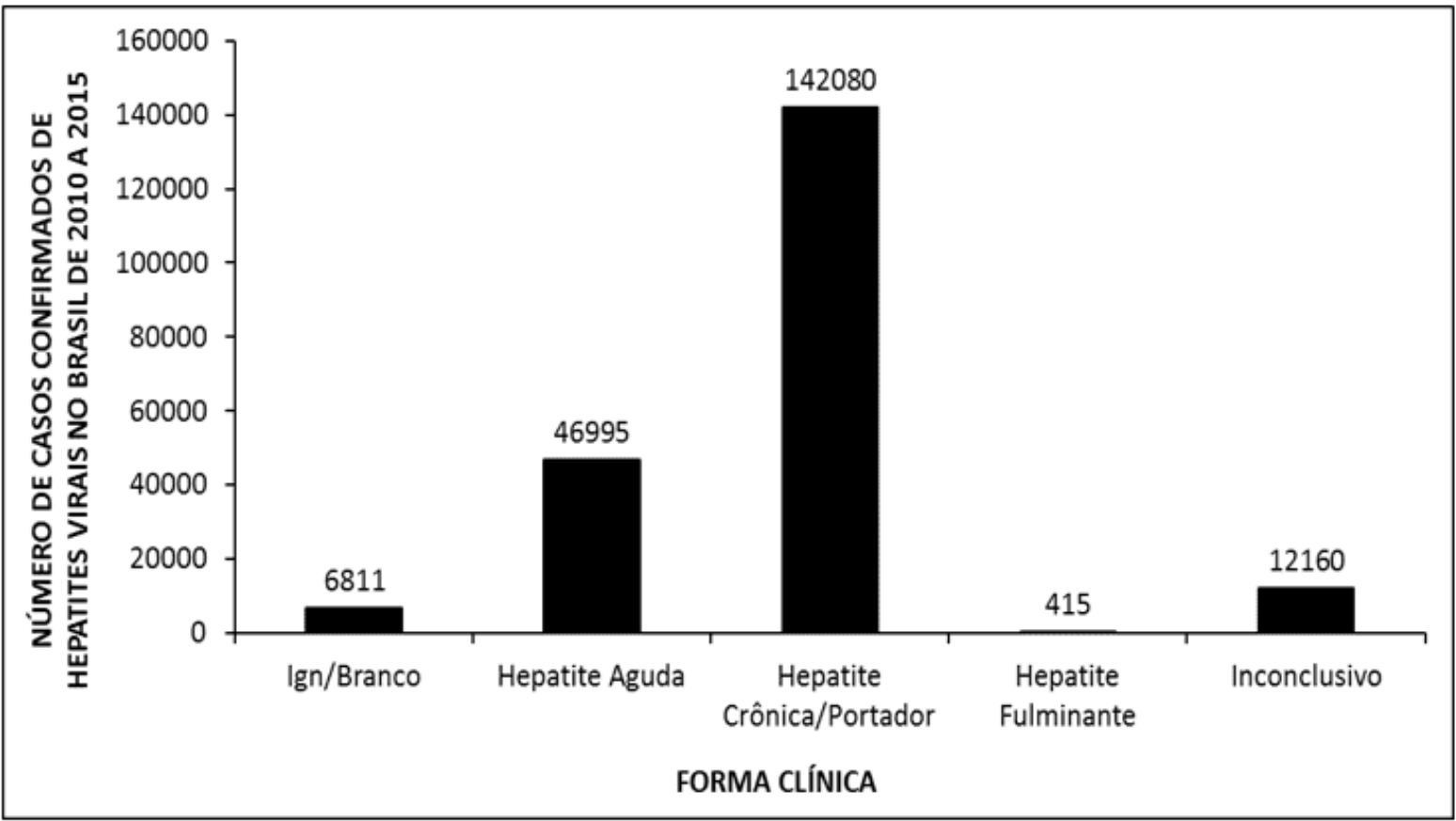

La figure 8 montre le nombre de cas confirmés d'hépatite virale au Brésil entre 2010 et 2015 par le niveau d'éducation des personnes infectées. Démontrer que le plus grand nombre de cas, lorsque la personne identifie sa scolarité, se produit chez les personnes ayant terminé leurs études secondaires et la deuxième avec la $5 \mathrm{e}$ à la $8 \mathrm{e}$ année incomplète de LA.

RC: 67661

Disponible en: https://www.nucleodoconhecimento.com.br/sante/dhepatite-virale 
Figure 8 Nombre de cas confirmés d'hépatite virale au Brésil entre 2010 et 2015 par la scolarisation des individus.

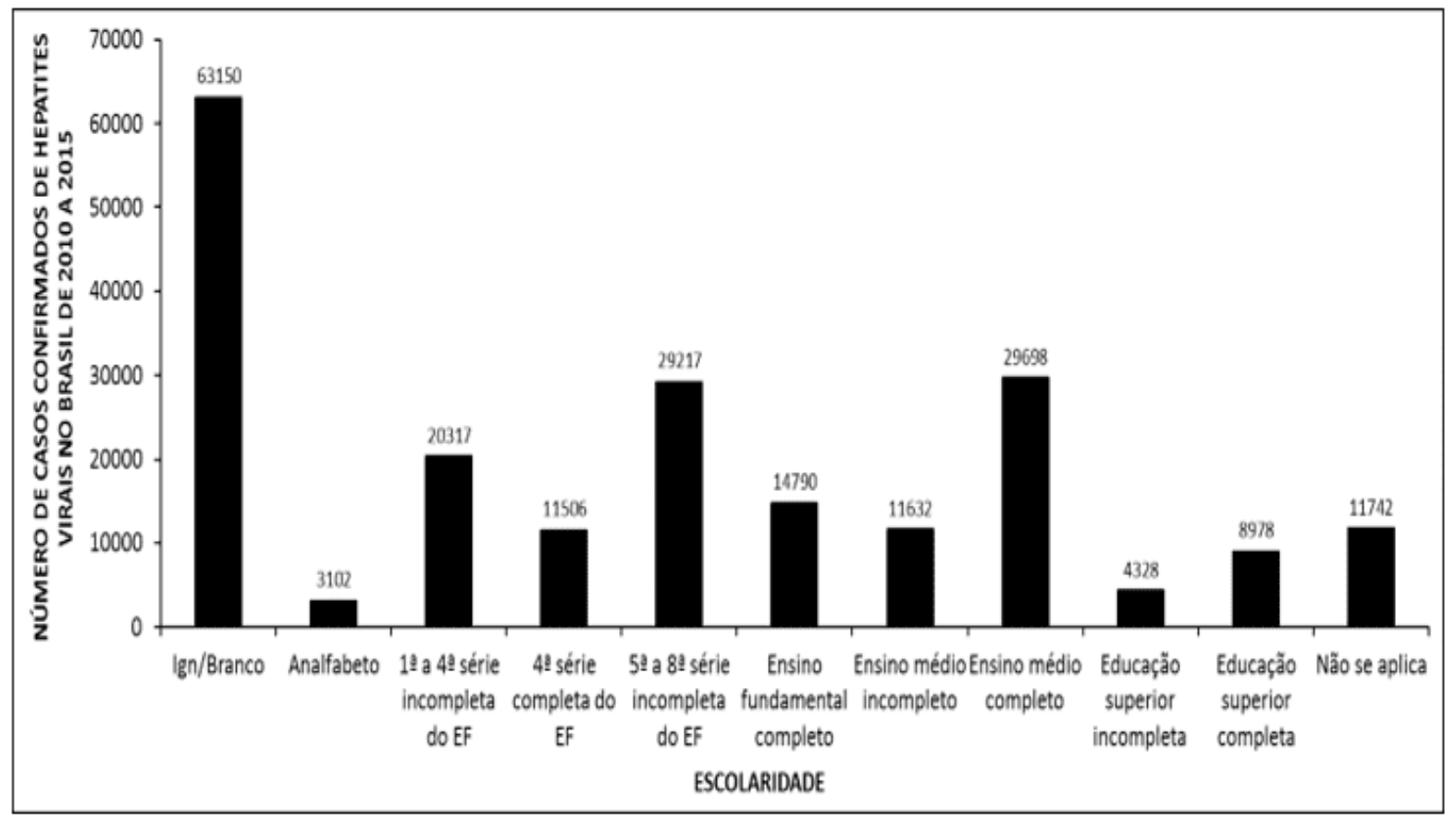

\section{DISCUSSION}

L'hépatite virale est un problème de santé publique complet au Brésil. En tenant compte de plusieurs facteurs, tels que l'irrégularité de la distribution des services de santé et l'inégalité concomitante des technologies diagnostiques, en plus des disparités économiques et sociales, il est possible de comprendre certaines des raisons qui sont liées au nombre élevé d'hépatites dans le pays. En outre, des connaissances inexactes sur le nombre de personnes infectées et une instruction insuffisante concernant les agents étiologiques de l'hépatite virale et leurs moyens de transmission contribuent également négativement à la dynamique de l'avancée de la pathologie au Brésil (FERREIRA et SILVEIRA, 2004).

Parmi les personnes infectées par les divers virus causant l'hépatite, la majorité est masculine. De nombreuses études indiquent que les hommes ont tendance à exercer moins de demande de services médicaux en général et, compte tenu de ce

RC: 67661

Disponible en: https://www.nucleodoconhecimento.com.br/sante/dhepatite-virale 
fait, souffrent plus de décès dus à des maladies chroniques (GOMES et al, 2007). Parmi les principaux groupes à risque d'infection par le virus de l'hépatite, en particulier le VHB, il y a : les professionnels de la santé, les personnes qui utilisent des médicaments intraveineux, les patients atteints d'hémodialyse, les professionnels du sexe et les hommes homosexuels (FERREIRA et SILVEIRA, 2004).

Grâce aux données du Bulletin épidémiologique sur l'hépatite virale de 2015, il est possible de vérifier les principales fourchettes d'âge dans lesquelles prédominent les cas d'hépatite. Dans le cas de l'hépatite A, l'infection a tendance à se produire principalement chez les enfants de moins de 10 ans, et le pic se situe entre 5 et 6 ans. En ce qui concerne l'hépatite $B$, le pic de cas se produit dans la fourchette d'âge de 20 et 30 ans. Dans le cas de l'hépatite $C$, il est à noter que le taux le plus élevé de cas survient chez les personnes âgées de 40 à 60 ans (BRASIL, 2015). Une observation importante est l'augmentation significative - 97,7\% - de la transmission de l'hépatite A chez les hommes, entre 20 et 39 ans, par des moyens sexuels, selon le Bulletin épidémiologique 2018 de l'hépatite virale (BRASIL, 2018a). La voie sexuelle de transmission de l'hépatite $C$ fait l'objet de débats, car, contrairement au potentiel reconnu de transmissibilité sexuelle de l'hépatite $\mathrm{B}$, le VHC a une infectiosité sexuelle qui se produit de façon limitée et, par conséquent, n'est pas en fait classée comme IST (Infection sexuellement transmissible) (ALMEIDA et MARTINS, 2015).

Au Brésil, entre 1999 et 2018, il y a eu 632814 cas confirmés d'hépatite virale. Parmi ces cas, 36,8 \% (233 027) sont liés à l'hépatite B, 36,1 \% (228 695) à l'hépatite $C$, qui représentent les chiffres les plus élevés. L'hépatite A représente $26,4 \%$ du total (167 108), en plus de $0,7 \%$ de l'hépatite $D$ (3 984), cette dernière étant l'hépatite ayant le plus faible nombre de notifications et une différence globale, en nombre, de l'autre. L'hépatite $C$, depuis 2015 , a dépassé le nombre annuel de taux d'incidence de l'hépatite $B$ (BRASIL, 2019). Un élément à prendre en compte est la co-infection par le virus de l'immunodéficience humaine $(\mathrm{VIH})$ et les hépatites $B(\mathrm{VHB})$ et $\mathrm{C}$ 
(VHC), puisque $70 \%$ à $90 \%$ des patients infectés par le VIH ont des marqueurs d'infection transmise par le VHB (FARIAS et al, 2012)

Le diagnostic clinique de l'hépatite virale se produit par des signes et des symptômes présentés par le patient, et les individus qui ont le virus(s) peuvent présenter des conditions symptomatiques aiguës, chroniques et même asymptomatiques. Dans l'hépatite aiguë, le patient peut se présenter avec le malaise, la fatigue, la nausée, la choluria (urine foncée), l'anorexie, l'ictère, et les selles blanchâtres-colorées. Dans le cas d'une image de l'hépatite chronique, parfois les individus peuvent être asymptomatiques, cependant, des participations de foie peuvent apparaître après une certaine période, telles que la cirrhose de foie, la fibrose hépatique et même le carcinome hépatocellulaire (selon l'évolution, d'autres organes peuvent être compromis). Le diagnostic se produit principalement dans la phase chronique de la maladie (principalement dans les hépatites $B$ et $C$ ). Au niveau du laboratoire, il est possible de détecter les marqueurs de l'hépatite, et le contenu utilisé pour ce processus sont les fluides oraux, le sang, le sérum ou le plasma du patient infecté, grâce à des techniques appelées immunoassays. Ces procédures visent à détecter l'antigène lui-même ou les anticorps et il existe différents types: analyses immunoenzymatiques, tests luminescents et rapides. En outre, il existe également des tests moléculaires, basés sur la réaction en chaîne de polyméseau (PCR) (BRASIL, 2018b et SBI, 2003)

La scolarisation est l'un des facteurs qui sont pris en compte épidémiologiquement pour comprendre l'impact de l'hépatite au Brésil. En ce qui concerne les cas confirmés d'hépatite virale, on observe qu'une grande proportion de personnes scolarisées de la cinquième à la huitième année du primaire, et de 1999 à 2018, chez les hommes, de tous les cas confirmés, étaient 21844 à correspondre à ce profil (inférieur uniquement à la catégorie « ignorée », selon les données du Bulletin épidémiologique sur l'hépatite virale 2019) (BRASIL, 2019).

RC: 67661

Disponible en: https://www.nucleodoconhecimento.com.br/sante/dhepatite-virale 


\section{CONCLUSIONS}

L'hépatite représente un vaste problème de santé publique au Brésil, et des facteurs tels que la répartition inégale des services de santé, les disparités socioéconomiques et d'autres problèmes contribuent à cet imbroglio.

Chez les personnes infectées, une grande partie est composée de personnes de sexe masculin, et la baisse visible de la demande de services de santé est un facteur important pour cette constatation. Pour cette raison, ils ont tendance à souffrir d'une participation plus chronique découlant du retard dans la recherche d'un professionnel de la santé. Les patients atteints d'hémodialyse, les travailleurs du sexe et les personnes qui utilisent des drogues injectables sont quelques-uns des principaux groupes à risque d'infection virale par l'hépatite. En outre, il a été constaté que, de 1999 à 2018, une grande proportion des cas confirmés d'hépatite (21 844 cas) correspondaient à des personnes dont la scolarité était incomplète de la cinquième à la huitième année de l'école primaire.

Les hépatites $B$ et $C$ sont les plus courantes chez les hépatites virales. Au cours des dernières années, il y a eu une augmentation significative (supérieure à 90\%) transmission de l'hépatite $B$ sexuellement. La transmission de l'hépatite C sexuellement est un plaisir, et son potentiel de transmissibilité sexuelle est considéré comme limité et n'est donc pas considéré comme une IST.

L'un des facteurs importants et des facteurs contribuant au taux de virus de l'hépatite infectés est leur co-infection par le $\mathrm{VIH}$. Environ $70 \%$ à $90 \%$ des patients séropositifs ont des marqueurs de l'infection antérieure au VHB.

Dans le cas de l'hépatite virale, les critères cliniques et de laboratoire sont pris en compte. Des signes et des symptômes tels que l'ictère, choluria, selles blanchâtres, fatigue, malaise, nausée, et d'autres peuvent se présenter dans le patient présentant I'hépatite. Cependant, il peut également y avoir des cas asymptomatiques, qui contribuent au retard du diagnostic et à la chronicité conséquente de la maladie. Des

$\mathrm{RC}: 67661$

Disponible en: https://www.nucleodoconhecimento.com.br/sante/dhepatite-virale 
tests de laboratoire (immunoassay, tests moléculaires) devraient être effectués pour détecter les marqueurs et déterminer l'agent étiologique à l'origine de la pathologie.

\section{RÉFÉRENCES}

ALMEIDA, C. S. C.; MARTINS, L. C. Soroepidemiologia do vírus da hepatite C em cônjuges de portadores desse vírus. Revista Paranaense de Medicina, v. 29, n.1, p. 11-16, 2015.

BRASIL. Ministério da Saúde. Portal da Saúde. PRENVENÇÃO-HEPATITES: HEPATITE D. Disponível em:< http://portalsaude.saude.gov.br/index.php/links-deinteresse/305-hepatites-virais/prevencao-hepatites/9124-hepatite-d> . 2014.

BRASIL. Ministério da Saúde. Portal da Saúde. PREnVEnçÃO-HEPATITES: HEPATITE B. Disponível em:<http://portalsaude.saude.gov.br/index.php/links-deinteresse/305-hepatites-virais/prevencao-hepatites/9130-hepatite-b > . 2014a.

BRASIL, 2015. Ministério da Saúde. Boletim Epidemiológico Hepatites virais. Ano IV, n. $01,2015$.

BRASIL, 2018a. Ministério da Saúde. Boletim Epidemiológico Hepatites Virais. V. 49, 2018.

BRASIL, 2018b. Ministério da saúde. Manual técnico para o diagnóstico das hepatites virais. 2018.

BRASIL, 2019. Ministério da Saúde. Boletim Epidemiológico Hepatites Virais. V.50, 2019.

FARIAS, N.; SOUZA, I.; COELHO, D. M.; OLIVEIRA, U. B.; BINELLI, C. A. Coinfecção pelos vírus das hepatites $B$ ou $C$ e da imunodeficiência adquirida: estudo exploratório no estado de São Paulo, Brasil, 2007 a 2010. Epidemiol. Serv. Saúde, v. 21 , n. 3, p. 475-486, 2012.

RC: 67661

Disponible en: https://www.nucleodoconhecimento.com.br/sante/dhepatite-virale 
FERREIRA, C. T.; SILVEIRA, T R. Hepatites virais: aspectos da epidemiologia e da prevenção. Rev. bras. epidemiol., v. 7, n. 4, p. 473-487, 2004 .

FERREIRA, A.R.; FAGUNDES, E.D.T.; QUEIROZ, T.C.N.; PIMENTA, J.R.; JÚNIOR, R.C. N.. Hepatites Virais A, B e C em crianças e adolescentes. Rev Med de Minas Gerais, (Supl 2): S46-S60, 2014.

FRANCISCO, P.M.S.B; DONALISIO, M.R; GABRIEL, F.J.O; BARROS, M.B.A. Vacinação contra hepatite $B$ em adolescentes residentes em Campinas, São Paulo, Brasil. REV BRAS EPIDEMIOL, 18(3): 552-567, JUL-SET 2015.

GOMES, R.; NASCIMENTO, E. F.; ARAÚJO, F. C. Por que os homens buscam menos os serviços de saúde do que as mulheres? As explicações de homens com baixa escolaridade e homens com ensino superior. Cad. Saúde Pública, v. 23, n.3, p. $565-574,2007$.

GUSMÃO, K.E et al. PERFIL CLÍNICO-EPIDEMIOLÓGICO DA HEPATITE C NA REGIÃO NORTE DO BRASIL ENTRE 2012 E 2015.Revistade Patologia do Tocantins, [S.I.], v. 4, n. 2, p. 41-45, jun. 2017.

LEITE, A.C.D. et al. . Avaliação das Internações Pela Hepatite Aguda B em Comparativo com a Aplicação de Doses da Vacina Contra Hbv, Na Região Norte. In: Luís Marcelo Aranha Camargo; Dionatas Ulises de Oliveira Meneguetti; Jader de Oliveira. (Org.). Atualidades em Medicina Tropical no Brasil: Educação em Saúde. 1ed.Rio Branco, Acre: Stricto Sensu Editora, 2020, p. 39-48.

MARGREITER, S. et al. Estudo de prevalêcia das hepatites virais b e c no município de palhoça-sc. Rev. Saíde Públ. Santa Cat., Florianópolis, v. 8, n. 2, p. 21-32, maio/ago. 2015.

MOCBEL, I.L.S.A. et al. CONHECIMENTO SOBRE HEPATITES A e E DOS ESTUDANTES DE UMA ESCOLA MUNICIPAL EM SANTARÉM - PA. Revista EM FOCO - Fundação Esperança/IESPES, [S.I.], v. 2, n. 24, p. 18-29, abr. 2016.

RC: 67661

Disponible en: https://www.nucleodoconhecimento.com.br/sante/dhepatite-virale 
NUNES, H.M. et al. Soroprevalência da infecção pelos vírus das hepatites $A B, C, D$ e E em município da região oeste do Estado do Pará, Brasil. Rev. Pan-Amaz. Saúde, Ananindeua, v. 7, n. 1, p. 55-62, mar. 2016.

SBI (SOCIEDADE BRASILEIRA DE INFECTOLOGIA). Boletim terapêutico de HIV/Aids, DTS e Hepatites Virais. Ano I, n. 4, 2003.

SILVA, C.M; VIANNA, G.S.P; SOARES, M.C.P; AMARAL, I.S.A.; MOIA, L.J.M.P. AVALIAÇÃO DO HEMOGRAMA EM PACIENTES TRATADOS PARA HEPATITE C1. Revista Paraense de Medicina - V.28 (2) abril-junho 2014.

Soumis : Novembre 2020.

Approuvé : Novembre 2020.

$\mathrm{RC}: 67661$

Disponible en: https://www.nucleodoconhecimento.com.br/sante/dhepatite-virale 